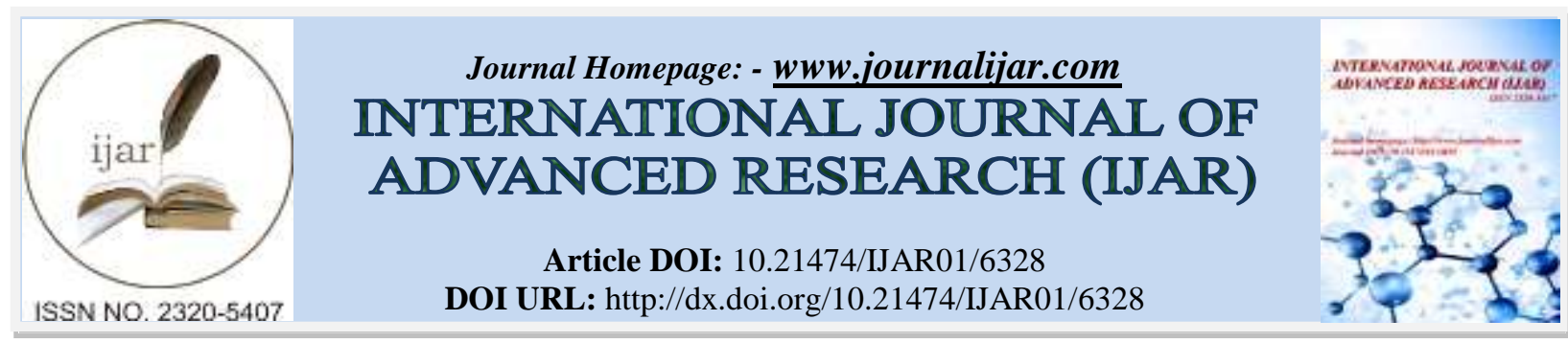

RESEARCH ARTICLE

\title{
SYNTHESIS AND BIOLOGICAL ACTIVITIES OF 1, 3, 4-OXADIAZOLE DERIVATIVES: A REVIEW OF LITERATURE.
}

Baijika P, Akash Marathakam, Midhula C C and Shadiha Saheed K.

Department of Pharmaceutical Chemistry, National College of Pharmacy, Manassery, Mukkam, Kozhikode 673602.

Manuscript Info

Manuscript History

Received: 17 November 2017

Final Accepted: 19 December 2017

Published: January 2018

Key words:-

1, 3, 4 oxadiazole, derivatives, synthesis,

biological activity.

\section{Abstract}

A series of derivatives of 1,3,4-oxadiazole having verities of biological activities can be synthesised by various methods. The activities include anticancer, antimicrobial, anti- inflammatory, antiHIV, anti tubercular, anti diabetic, antifungal etc. In this article we have summarized various methods for synthesis of derivatives of 1,3 , 4-oxadiazole nucleus and evaluation of various biological activities.

\section{Introduction:-}

Simple five membered heterocycles possessing one oxygen atom and two nitrogen atoms are considered as 1, 3, 4oxadiazole and its derivatives. 1,3,4-oxadiazole can exist in different isomeric forms, they are 1,2,5-oxadiazole(fig: 1), 1,2,4-oxadiazole(fig: 2), 1,2,3-oxadiazole(fig: 3) and 1,3,4-oxadiazole(fig: 4). Oxygen and nitrogen containing five member heterocyclic nucleus is assessed against various diseases. So they are given importance in medicinal chemistry because of their diverse medicinal potential. Substituted 1, 3, 4-oxadiazole is have broad spectrum of biological activities in pharmaceutical and agrochemical field. ${ }^{[8]}$<smiles>c1cnon1</smiles>

(fig: 1)<smiles>c1ncon1</smiles>

(fig: 2)<smiles>c1conn1</smiles>

(fig: 3)<smiles>c1nnco1</smiles>

(fig: 4)

1, 3, 4-oxadiazole shows wide variety of activities such as virucidal, CNS depressant, genotoxic, anticonvulsant, insecticidal, anti-tubercular, Anti-HIV, herbicidal, anti-inflammatory. It is also known to exhibit anti malarial, Muscle relaxants, anti tumour, lipid peroxidation inhibitor, antimicrobial, and remarkable analgesic, anticonvulsant, diuretic, hypnotic and sedative properties. There for 1,3,4-oxadiazole is commonly used in the area of new drug development. ${ }^{[5]}$

For the synthesis of 1,3,4-oxadiazole the conventional method used involve the intermolecular condensation of acid hydrazide with carboxylic acid in presence of cyclising agents such as phosphorous oxy chloride, polyphosohoric acid, acetic anhydride. Another reaction involves the condensation with carbon disulfide, potassium hydroxide and ethanol. In this reaction thiol substituted 1, 3, 4-oxadiazole is formed. ${ }^{[10]}$

This article contains review of the work done by different authors on the synthesis and evaluation of biological activity of 1, 3, 4-oxadiazole.

Corresponding Author:- Baijika p.

Address:- Department Of Pharmaceutical Chemistry, National College Of Pharmacy, Manassery, 


\section{Synthesis and biological activity of $1,3,4$-oxadiazole:-}

Gudipati R, et al., ${ }^{[4]}$ (2011) were synthesised 5- or 7-substituted 3-\{4-(5-mercapto-1,3,4-oxadiazol-2yl)phenylimino -indolin-2-one derivatives (scheme: 1 ) having anticancer activity by treating 5 -(4-aminophenyl)1,3,4-oxadiazole-2-thiol with different isatin derivatives.

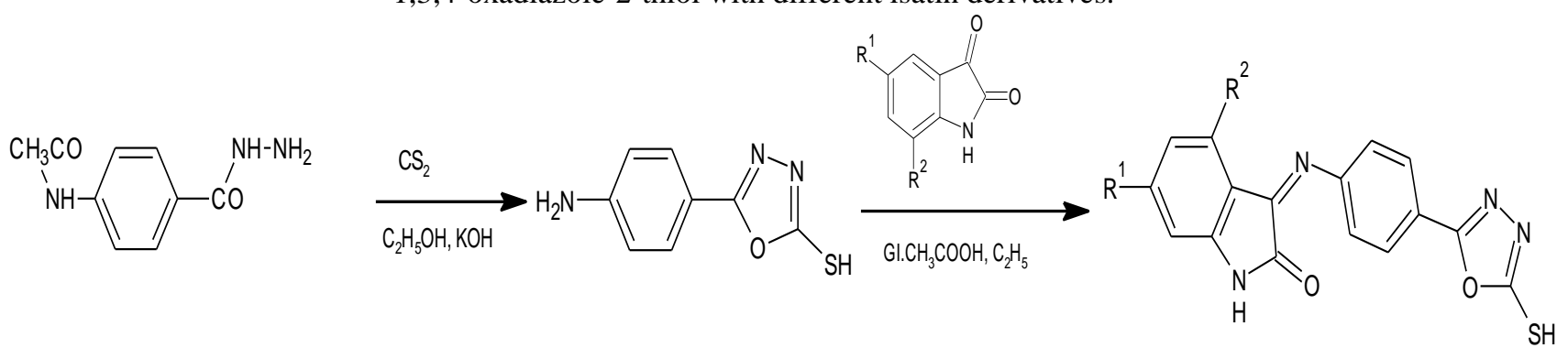

$$
\begin{gathered}
\mathrm{R}^{1}=\mathrm{H}, \mathrm{F}, \mathrm{Cl}, \mathrm{Br}, \mathrm{CH}_{3}, \mathrm{NO}_{2}, \mathrm{COOH} \\
\mathrm{R}^{2}=\mathrm{H}, \mathrm{Cl}, \mathrm{NO}_{2}, \mathrm{CH}_{3}, \mathrm{COOH}, \mathrm{COOCH} \mathrm{COOH}_{3}
\end{gathered}
$$

\section{Scheme 1:-}

Ahsan MJ, et al. ${ }^{[1]}$ (2011) were synthesised a series of 1,5-dimethyl-2-phenyl-4-\{[(5-aryl-1,3,4-oxadiazol-2yl)methyl]amino -1,2-dihydro-3H-pyrazol-3-one derivatives (scheme: 2) having antimicrobial and antitubercular activity.<smiles>Cc1c(N)c(=O)n(-c2ccccc2)n1C</smiles>
$\mathrm{Ar}$ = 2-chlorophenyl, 4-pyridinyl, 4-aminophenyl, 2-hydroxyphenyl, 4-methyl phenyl benzyl

\section{Scheme 2:-}

Kotaiah Y, et al., ${ }^{[7]}$ (2012) were synthesised N-substituted phenyl-5-methyl-6-(5-(4-substitutedphenyl)-1,3,4oxadiazol-2-yl) thieno [2,3-d]pyrimidin-4-amine derivatives (scheme: 3) and phenylamino-5-methylthieno[2,3d]pyrimidine-6-carboxylic acid derivatives were substituted on it. In this 4 derivatives having antioxidant activity.

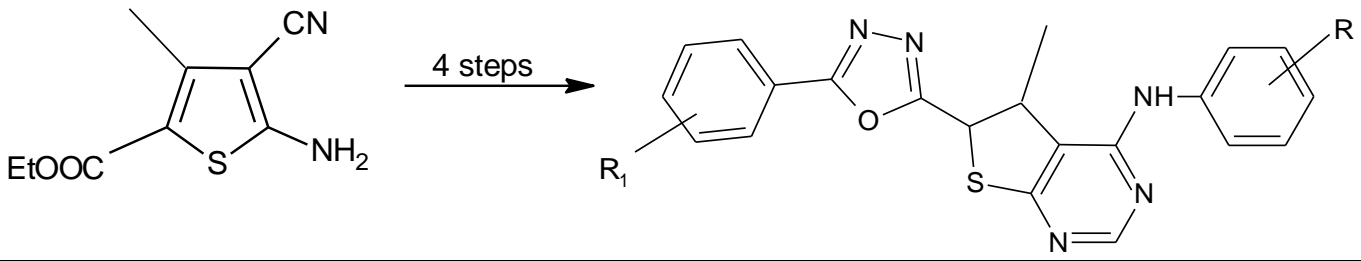

\begin{tabular}{|c|c|c|c|c|c|c|c|c|c|}
\hline $\mathrm{R}_{1}$ & $4-\mathrm{Cl}$ & $4-\mathrm{Cl}$ & $4-\mathrm{F}$ & $4-\mathrm{F}$ & $3-\mathrm{Cl}, 4-\mathrm{F}$ & $3-\mathrm{Cl}, 4-\mathrm{F}$ & $3-\mathrm{Cl}, 4-\mathrm{F}$ & 2,4-di-F & 2,4-di-F \\
\hline $\mathrm{R}$ & $4-\mathrm{H}$ & $4-\mathrm{Cl}$ & $4-\mathrm{Cl}$ & $4-\mathrm{NO}_{2}$ & $4-\mathrm{H}$ & $4-\mathrm{NO}_{2}$ & $4-\mathrm{Cl}$ & $4-\mathrm{Cl}$ & $4-\mathrm{NO}_{2}$ \\
\hline
\end{tabular}

Scheme 3:-

Ramazani A, et al., ${ }^{[14]}$ (2011) were synthesised 1,3,4-oxadiazole derivatives (scheme: 4) by the reaction between (N-isocyanimino) triphenylphosphorane, bis-aldehydes (isophthalaldehyde and terphthalaldehyde) and aromatic (or heteroaromatic) carboxylic acids. 


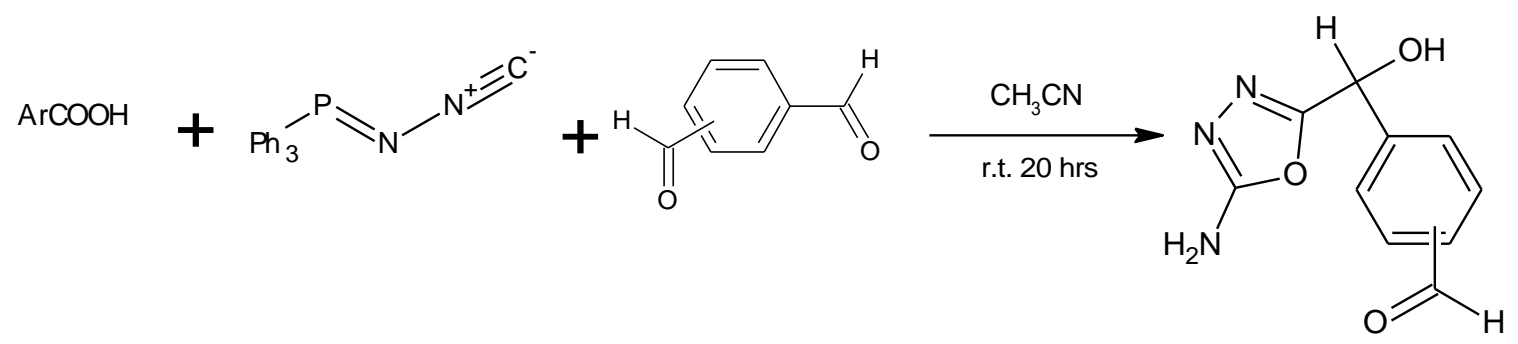

$\mathrm{Ar}=$ aromatic carboxylic acids and aromatic bis aldehyde

\section{Scheme 4:-}

$\mathrm{Xu}$ WM, et al., ${ }^{[22]}$ (2012) were designed and synthesised 1,3,4-oxadiazole moiety containing sulfone groups (scheme: 5) and were reported to have ability to mycelia growth of Ralstonia solanacearum in vitro also having better control effect against tobacco bacterial wilt so sulfone derivatives containing 1,3,4-oxadiazole can be used to develop potential bactericides for plants.

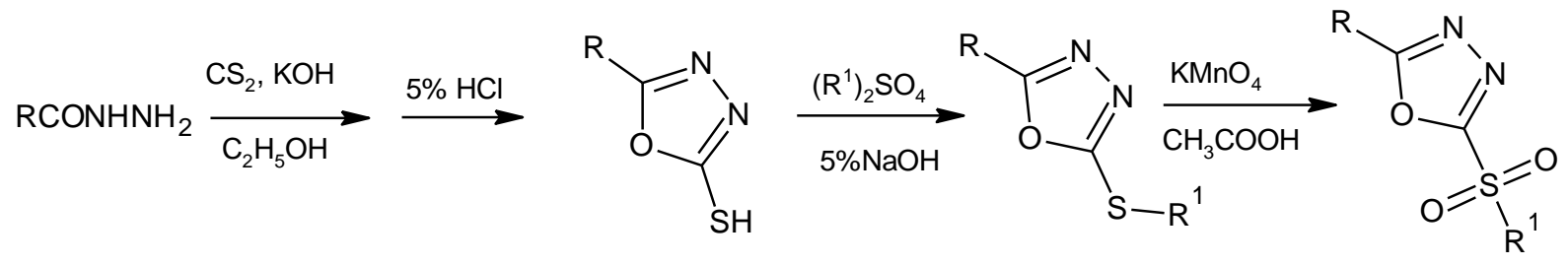

$\mathrm{R}=$ benzoic acid, fatty acids, heterocyclic acids $\mathrm{R}^{1}=\mathrm{CH}_{3}, \mathrm{CH}_{2} \mathrm{CH}_{3}$

Scheme 5:-

Puthiyapurayil P, et al., ${ }^{[12]}$ (2012), were synthesised S-substituted-1, 3, 4-oxadiazole bearing N-methyl4 (trifluoromethyl)phenyl pyrazole moiety (scheme: 6) and reported to have anticancer activity.

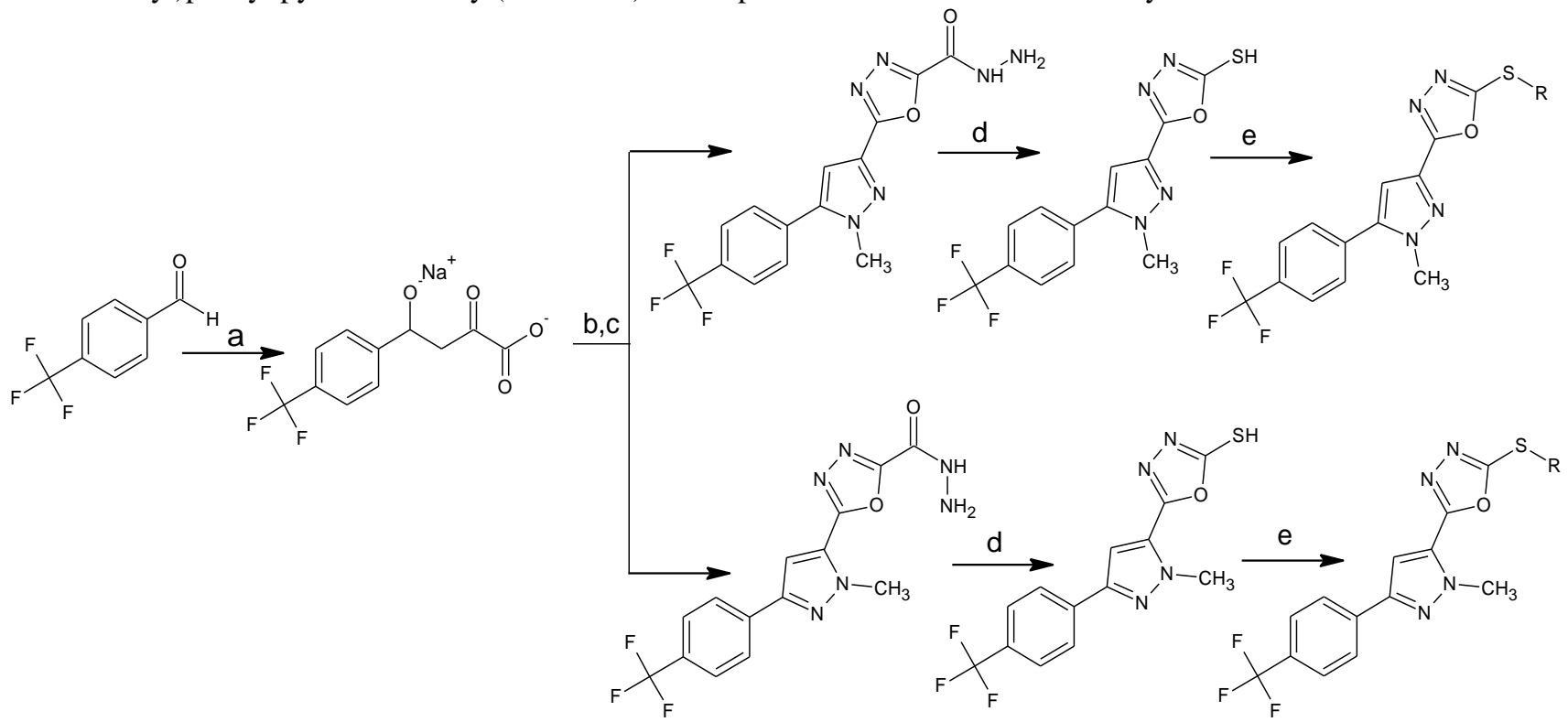

Reagents and conditions: (a) Dimethyl oxalate, $\mathrm{NaOMe}, \mathrm{MeOH}, \mathrm{RT}$; (b) Methyl hydrazine hydrate, Gla. AcOH, 105 ${ }^{0} \mathrm{C}$; (c) NH4. H2O, MeOH, 100C; (d) $\mathrm{CS}_{2}, \mathrm{KOH}, \mathrm{MeOH}$, Reflux; (e) ReX, K2CO3, DMF, $80{ }^{\circ} \mathrm{C}$.

\section{$\mathrm{R}=$ substituted aryl halides}

Scheme 6:-

Zhang XM, et al., ${ }^{[23]}$ (2011), were synthesised 1, 3, 4-oxadiazole derivative condensed with 1,4-benzodioxan moiety and some of the derivatives (scheme: 7) were having antitumor activity. 


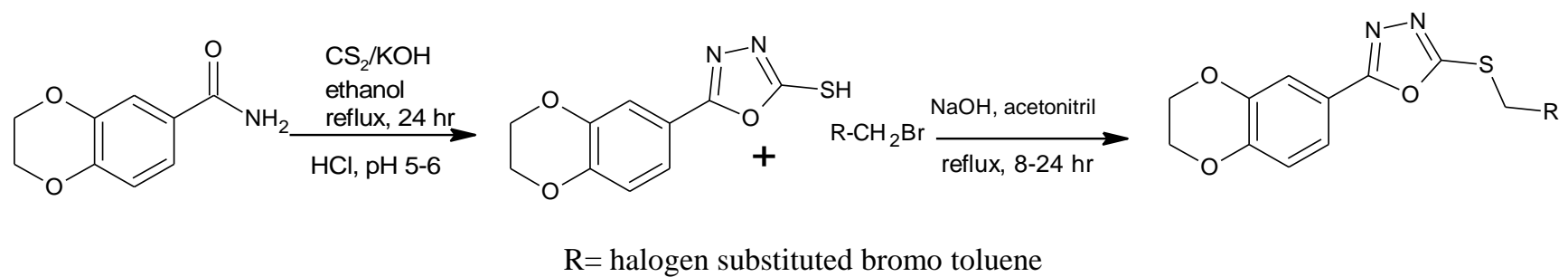

Scheme 7:-

Sun J, et al., ${ }^{[19]}$ (2013) were synthesised quinoline derivatives of 1, 3, 4-oxadiazole (scheme: 8) and were having anticancer activity.

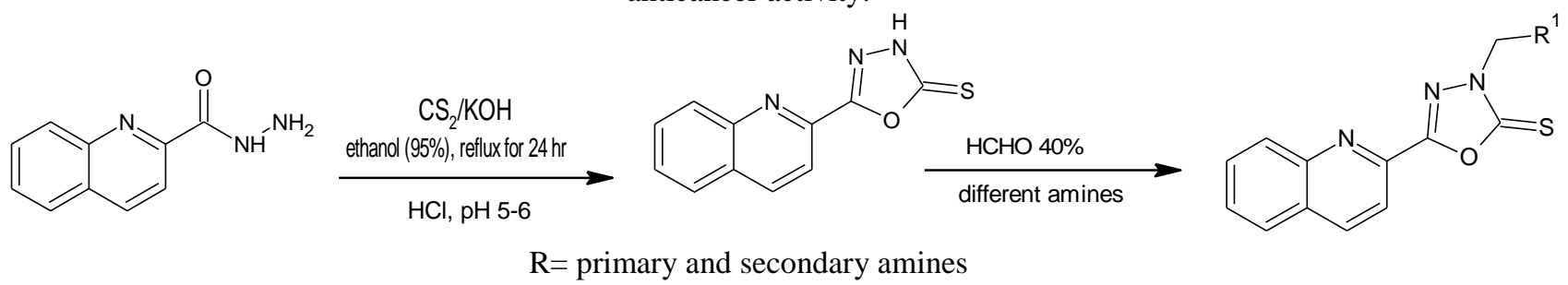

\section{Scheme 8:-}

Rane RA, et al., ${ }^{[15]}$ (2013) were synthesised 42 novel 4-nitropyrrole-based 1, 3, 4-oxadiazoles (scheme: 9) and evaluated for anti-bacterial, anti-fungal and anti-tubercular activities.<smiles>[R]c1nnc(-c2cc([N+](=O)[O-])cn2[R]([O-])([O-])O)o1</smiles>

$$
\begin{aligned}
& \mathrm{R}=\mathrm{H}, \mathrm{CH}_{3} \\
& \mathrm{R}=\text { aryl, heteroaryl }
\end{aligned}
$$

\section{Scheme 9:-}

Valente S, et al., ${ }^{[21]}$ (2014) were reported 1, 3, 4-oxadiazole (scheme: 10) containing hydroxamates and 2aminoanilides as histone deacetylase inhibitors. In this some of them were most potent and selective against HDAC.

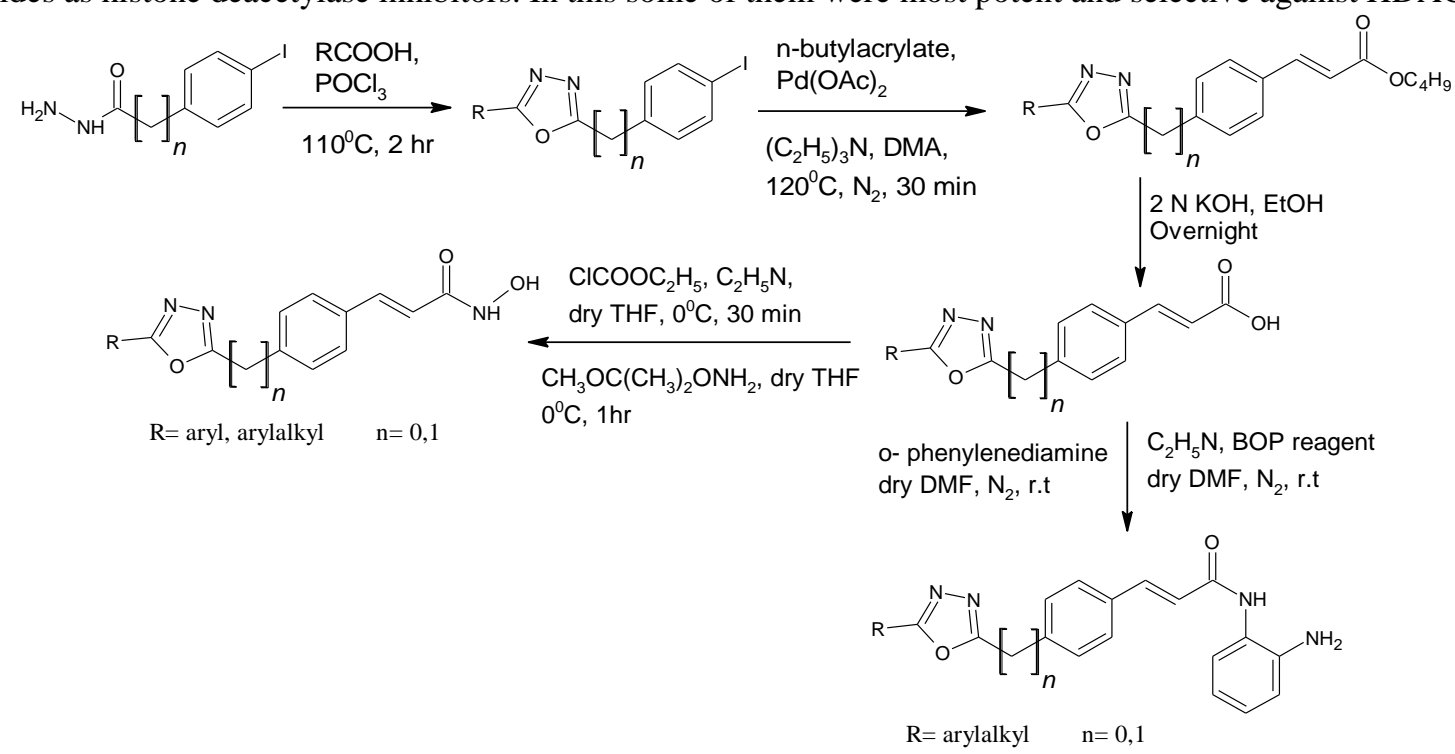




\section{Scheme 10:-}

Lai $\mathrm{H}$, et al., ${ }^{[9]}$ (2012) were synthesised a series of functionalized 1,2-benzisothiazol-3(2H)-one-1,3,4-oxadiazole hybrid derivatives (scheme: 11) and screened against Dengue and West Nile virus proteases.<smiles>O=C(O)C([Tl])n1sc2ccccc2c1=O</smiles><smiles>[R]C(=O)NNCC</smiles><smiles>NC(=O)C([Tl])n1sc2ccccc2c1=O</smiles>

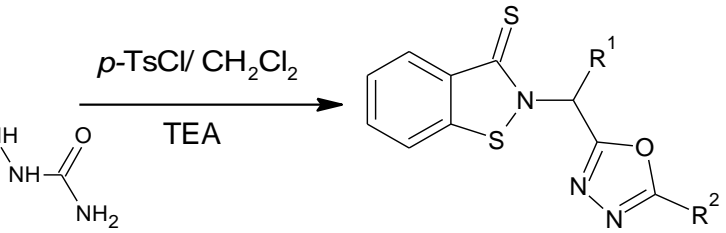

$\mathrm{R}^{1}=$ methyl, benzyl, phenethyl, $\mathrm{m}$ - flurobenzyl

$\mathrm{R}^{2}=$ substituted phenyl

\section{Scheme 11:-}

Khan KM, et al., ${ }^{[6]}$ (2013) were synthesised, a series of 5-substituted-1,3,4-oxadiazole-2yl-N-(2 methoxy-5chlorophenyl)-2-sulfanyl acetamide (scheme: 12) and reported their activity against acetyl cholinesterase.<smiles>[R]C(=O)NNNc1ccc(O)c(NC(=O)CBr)c1</smiles>

$\mathrm{R}=$ substituted aryl, heteroaryl

Scheme 12:-

El-Din MM, et al. ${ }^{[3]}$ (2014) were reported the synthesis of a new series of 1,3,4-oxadiazole derivatives possessing sulfonamide moiety (scheme: 13) having in vitro antiproliferative activities against NCI-58 human cancer cell lines of nine different cancer types.

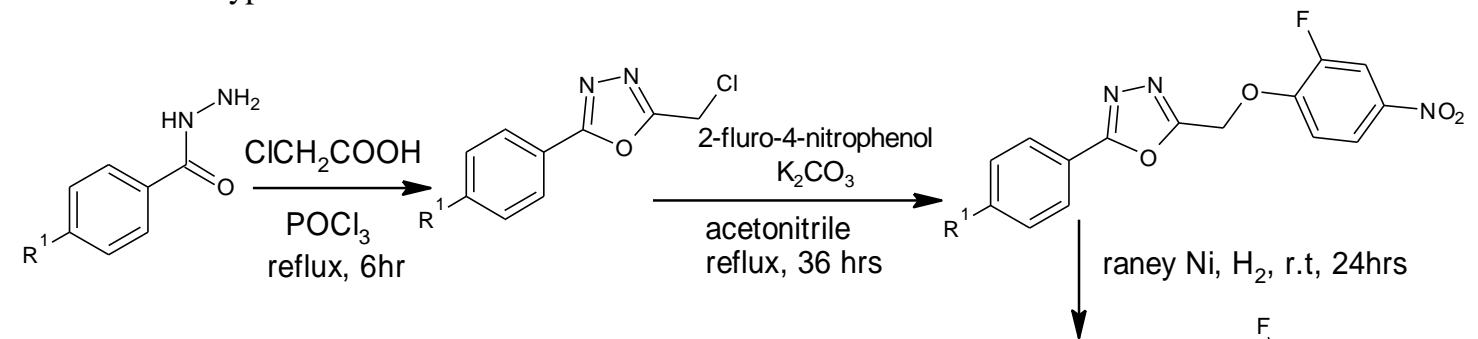

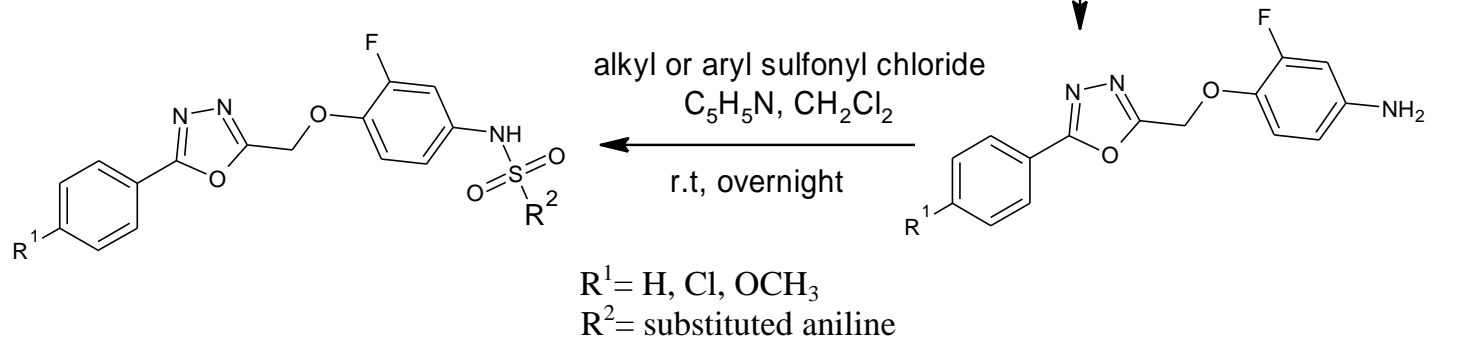

Scheme 13:-

Singh AK, et al., ${ }^{[18]}$ (2013) were synthesised a series of 1, 3, 4- oxadiazole derivatives (scheme: 14) and evaluated for anti-inflammatory activity. 


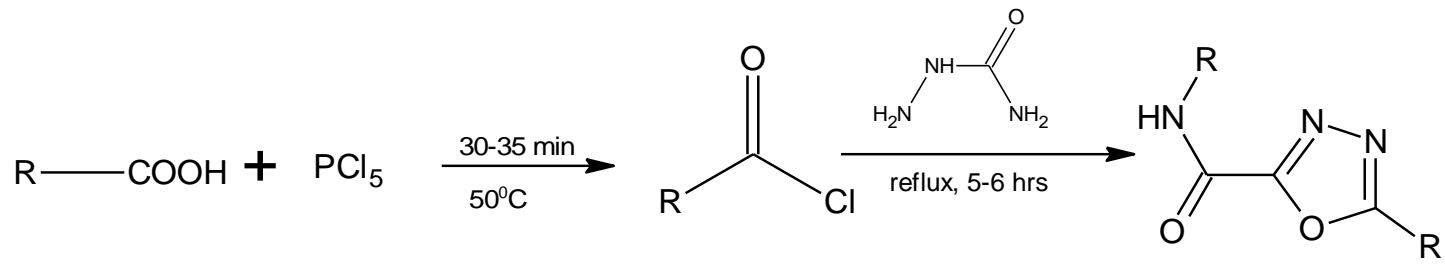

$\mathrm{R}=$ substituted phenyl

Scheme 14:-

Tabatabai SA, et al., ${ }^{[20]}$ (2013) were synthesised some derivatives of 2-(2-Phenoxy) phenyl-1, 3, 4-oxadiazole (scheme: 15) and were reported as benzodiazepine receptor agonists.

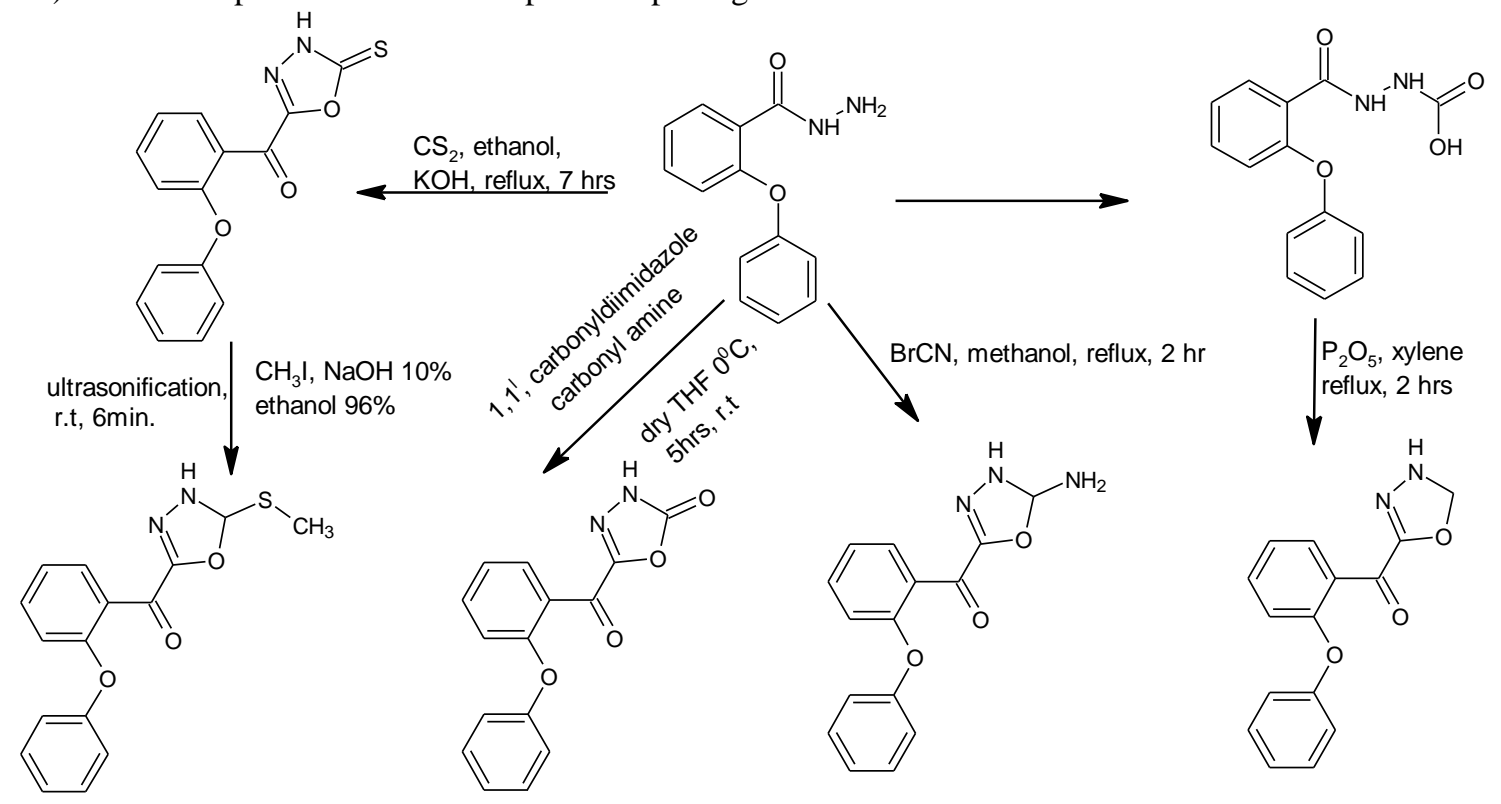

Scheme 15:-

Desai NC, et al., ${ }^{[2]}$ YM (2014) were synthesised novel series of 2-\{5-[4-(1-aza-2-(2-thienyl) vinyl ) phenyl](1,3,4oxa-diazol-2-ylthio) $\}$-N-arylacetamides (scheme: 16) and screened for their antibacterial and antifungal activities.

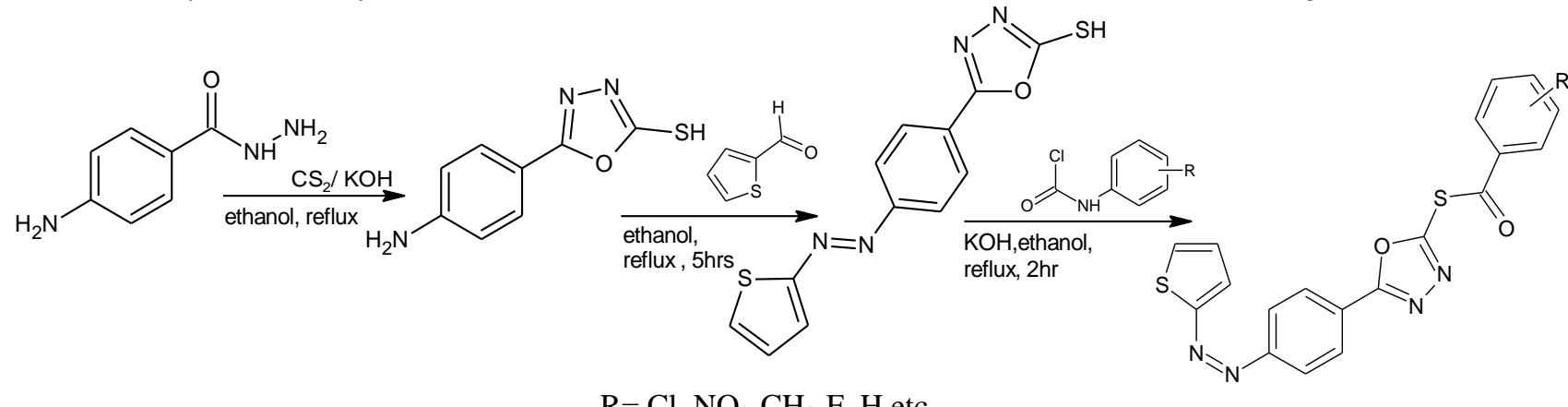

$\mathrm{R}=\mathrm{Cl}, \mathrm{NO}_{2}, \mathrm{CH}_{3}, \mathrm{~F}, \mathrm{H}$ etc

Scheme 16:-

Raval JP, et al., ${ }^{[16]}$ (2011) were synthesised a series of 2(4-pyridyl)-5[(aryl/heteroarylamino)-1-oxoethyl]thio-1,3,4oxadiazole (scheme: 17) and evaluated for antibacterial activity. 


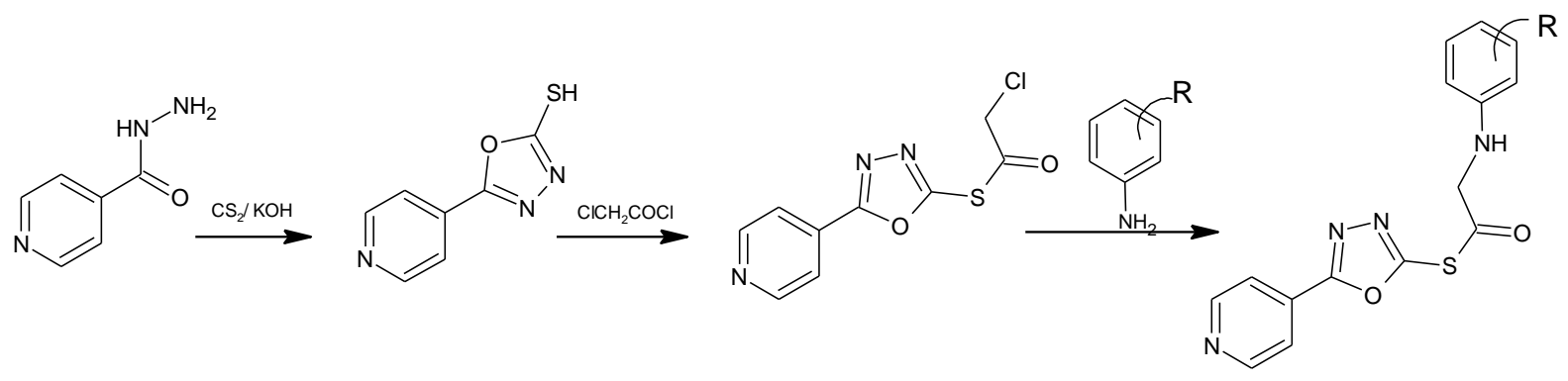

$\mathrm{R}=\mathrm{H}, \mathrm{Cl}, \mathrm{NO}_{2}, \mathrm{CH}_{3}$ etc

\section{Scheme 17:-}

Ningaiah S, et al., ${ }^{[11]}$ (2014) were synthesised a novel series of 2-(5-methyl-1,3-diphenyl-1H-pyrazol-4-yl)-5phenyl-1,3,4-oxadiazoles (scheme: 18) and evaluated for antimicrobial activity.

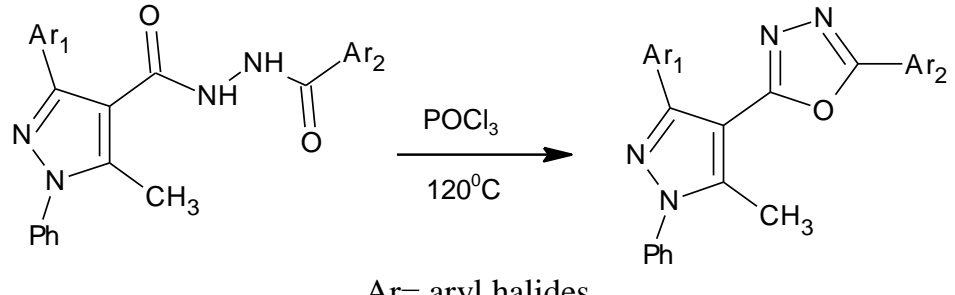

Scheme 18:-

Qi DQ, et al., ${ }^{[13]}$ (2015) were designed and synthesised a series of pyrazole-based 1, 3, 4-oxadiazole derivatives (scheme: 19). The fluorescence properties of all the compounds were analysed in dimethyl sulfoxide media and were evaluated for their $n$ vitro inhibitory activity against commercial enzyme xanthine oxidase (XO) by measuring the formation of uric acid from xanthine.
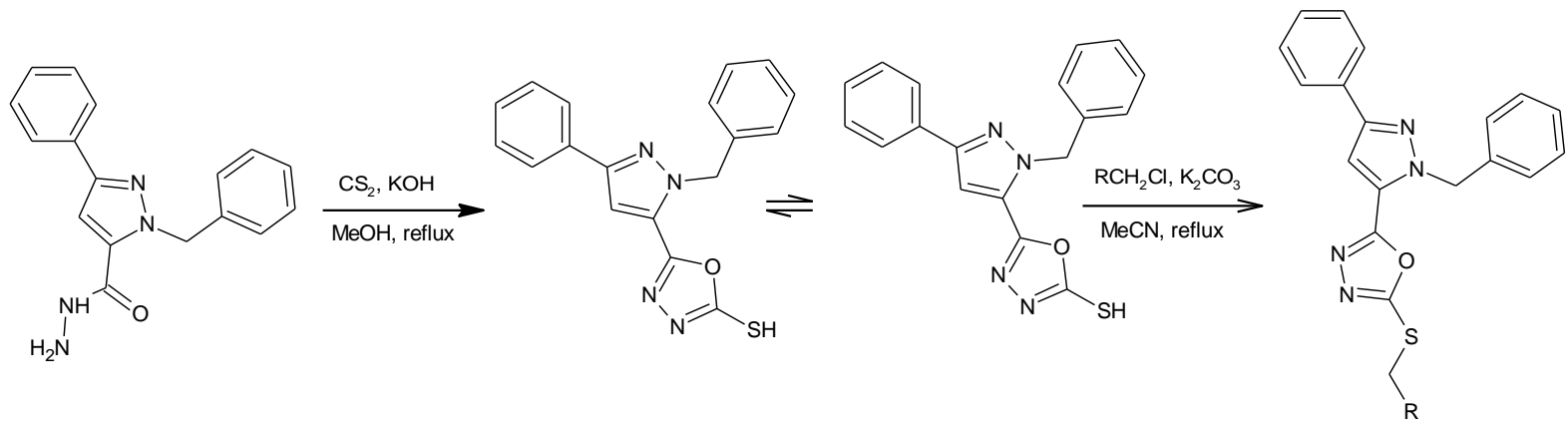

$\mathrm{R}=4-\mathrm{FC}_{6} \mathrm{H}_{4}, 4-\mathrm{ClC}_{6} \mathrm{H}_{4}, 4-\mathrm{BrC}_{6} \mathrm{H}_{4}, 4-\mathrm{NO}_{2} \mathrm{C}_{6} \mathrm{H}_{4}, 4-\mathrm{CNC}_{6} \mathrm{H}_{4}, 4-\mathrm{CH}_{3} \mathrm{C}_{6} \mathrm{H}_{4}, 3-\mathrm{FC}_{6} \mathrm{H}_{4}, 3-\mathrm{ClC}_{6} \mathrm{H}_{4}, 3-\mathrm{BrC}_{6} \mathrm{H}_{4}, 3-\mathrm{NO}_{2} \mathrm{C}_{6} \mathrm{H}_{4}$, $\mathrm{CH}\left(\mathrm{CH}_{3}\right)_{2} \mathrm{C}_{6} \mathrm{H}_{4}, \mathrm{COOCH}_{2} \mathrm{CH}_{3} \mathrm{C}_{6} \mathrm{H}_{4}$

\section{Scheme 19:-}

Roy PP, et al., ${ }^{[17]}$ (2017) were synthesised some novel 2, 5- Disubstituted 1, 3, 4-Oxadiazole derivatives (scheme: 20) using different aromatic benzaldehyde, and evaluated for their anticancer activity against Ehrlich Ascites Carcinoma (EAC) bearing albino mice. 

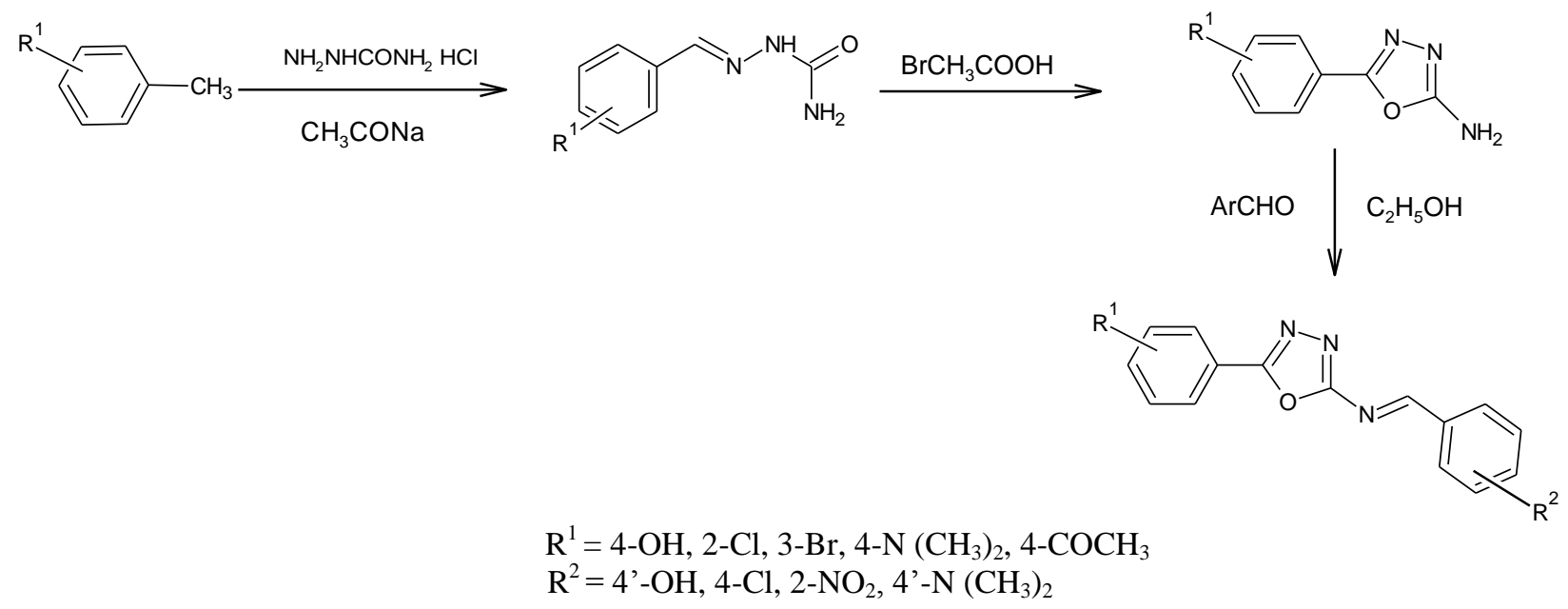

Scheme 20:-

\section{Conclusion:-}

This article summarizes the synthesis and biological activities of 1, 3, 4-oxadiazole derivatives. From this it is found that this five member heterocyclic molecule can be synthesised by various methods and those derivatives are having verities of activities. Such as anticancer, antimicrobial, anti- inflammatory, anti- HIV, anti tubercular, anti diabetic, antifungal etc. So study on this molecule is useful to the mankind.

\section{Bibliography:-}

1. Ahsan MJ, Samy JG, Khalilullah H, Nomani MS, Saraswat P, Gaur R, Singh A. Molecular properties prediction and synthesis of novel 1, 3, 4-oxadiazole analogues as potent antimicrobial and antitubercular agents. Bioorganic \& medicinal chemistry letters. 2011 Dec 15;21(24):7246-50.

2. Desai NC, Dodiya AM, Rajpara KM, Rupala YM. Synthesis and antimicrobial screening of 1, 3, 4-oxadiazole and clubbed thiophene derivatives. Journal of Saudi Chemical Society. 2014 Jul 31;18(3):255-61.

3. El-Din MM, El-Gamal MI, Abdel-Maksoud MS, Yoo KH, Oh CH. Synthesis and in vitro antiproliferative activity of new 1, 3, 4-oxadiazole derivatives possessing sulfonamide moiety. European journal of medicinal chemistry. 2015 Jan 27;90:45-52.

4. Gudipati R, Anreddy RN, Manda S. Synthesis, characterization and anticancer activity of certain 3-\{4-(5mercapto-1, 3, 4-oxadiazole-2-yl) phenylimino $\}$ indolin-2-one derivatives. Saudi Pharmaceutical Journal. 2011 Jul 31;19(3):153-8.

5. Kavitha S, Kannan K, Gnanavel S. Synthesis, characterization and biological evaluation of novel 2, 5 substituted-1, 3, 4 oxadiazole derivatives. Saudi Pharmaceutical Journal. 2017 Mar 31;25(3):337-45.

6. Khan KM, Ashraf M, Ahmad I, Ejaz SA. Synthesis, characterization and biological screening of 5-substituted1, 3, 4-oxadiazole-2yl-N-(2-methoxy-5-chlorophenyl)-2-sulfanyl acetamide. Pak. J. Pharm. Sci. 2013 Mar;26(2):345-52.

7. Kotaiah Y, Harikrishna N, Nagaraju K, Rao CV. Synthesis and antioxidant activity of 1, 3, 4-oxadiazole tagged thieno [2, 3-d] pyrimidine derivatives. European Journal of Medicinal Chemistry. 2012 Dec 31;58:340-5.

8. Kumar KA, Jayaroopa P, Kumar GV. Comprehensive review on the chemistry of 1, 3, 4-oxadiazoles and their applications. International Journal of ChemTech Research. 2012;4(4):1782-91.

9. Lai H, Dou D, Aravapalli S, Teramoto T, Lushington GH, Mwania TM, Alliston KR, Eichhorn DM, Padmanabhan R, Groutas WC. Design, synthesis and characterization of novel 1, 2-benzisothiazol-3 (2H)-one and 1, 3, 4-oxadiazole hybrid derivatives: potent inhibitors of Dengue and West Nile virus NS2B/NS3 proteases. Bioorganic \& medicinal chemistry. 2013 Jan 1;21(1):102-13.

10. Modi V, Modi P. Oxadiazole: Synthesis, characterization and biological activities. Journal of Saudi Chemical Society. 2012 Jul 31;16(3):327-32.

11. Ningaiah S, Bhadraiah UK, Doddaramappa SD, Keshavamurthy S, Javarasetty C. Novel pyrazole integrated 1, 3, 4-oxadiazoles: Synthesis, characterization and antimicrobial evaluation. Bioorganic \& medicinal chemistry letters. 2014 Jan 1;24(1):245-8. 
12. Puthiyapurayil P, Poojary B, Chikkanna C, Buridipad SK. Design, synthesis and biological evaluation of a novel series of 1, 3, 4-oxadiazole bearing N-methyl-4-(trifluoromethyl) phenyl pyrazole moiety as cytotoxic agents. European journal of medicinal chemistry. 2012 Jul 31;53:203-10.

13. Qi DQ, Yu CM, You JZ, Yang GH, Wang XJ, Zhang YP. Synthesis, crystal structures, fluorescence and xanthine oxidase inhibitory activity of pyrazole-based 1, 3, 4-oxadiazole derivatives. Journal of Molecular Structure. 2015 Nov 15;1100:421-8.

14. Ramazani A, Ahmadi Y, Mahyari A. One-pot efficient synthesis of fully substituted 1, 3, 4-oxadiazole derivatives from ( $\mathrm{N}$-isocyanimino) triphenylphosphorane, carboxylic acids, and aromatic bis-aldehydes. Synthetic Communications. 2011 Aug 1;41(15):2273-82.

15. Rane RA, Bangalore P, Borhade SD, Khandare PK. Synthesis and evaluation of novel 4-nitropyrrole-based 1, 3, 4-oxadiazole derivatives as antimicrobial and anti-tubercular agents. European journal of medicinal chemistry. 2013 Dec 31;70:49-58.

16. Raval JP, Akhaja TN, Jaspara DM, Myangar KN, Patel NH. Synthesis and in vitro antibacterial activity of new oxoethylthio-1, 3, 4-oxadiazole derivatives. Journal of Saudi Chemical Society. 2014 Apr 30;18(2):101-6.

17. Roy PP, Bajaj S, Maity TK, Singh J. Synthesis and Evaluation of Anticancer Activity of 1, 3, 4-Oxadiazole Derivatives against Ehrlich Ascites Carcinoma Bearing Mice and Their Correlation with Histopathology of Liver. receptor. 2017 Apr 1;15:16.

18. Singh AK, Lohani M, Parthsarthy R. Synthesis, characterization and anti-inflammatory activity of some 1, 3, 4oxadiazole derivatives. Iranian journal of pharmaceutical research: IJPR. 2013;12(2):319.

19. Sun J, Zhu H, Yang ZM, Zhu HL. Synthesis, molecular modeling and biological evaluation of 2-aminomethyl5-(quinolin-2-yl)-1, 3, 4-oxadiazole-2 (3H)-thione quinolone derivatives as novel anticancer agent. European journal of medicinal chemistry. 2013 Feb 28;60:23-8.

20. Tabatabai SA, Lashkari SB, Zarrindast MR, Gholibeikian M, Shafiee A. Design, synthesis and anticonvulsant activity of 2-(2-phenoxy) phenyl-1, 3, 4-oxadiazole derivatives. Iranian journal of pharmaceutical research: IJPR. 2013;12(Suppl):105.

21. Valente S, Trisciuoglio D, De Luca T, Nebbioso A, Labella D, Lenoci A, Bigogno C, Dondio G, Miceli M, Brosch G, Del Bufalo D. 1, 3, 4-Oxadiazole-containing histone deacetylase inhibitors: anticancer activities in cancer cells. Journal of medicinal chemistry. 2014 Jul 8;57(14):6259-65.

22. Xu WM, Han FF, He M, Hu DY, He J, Yang S, Song BA. Inhibition of tobacco bacterial wilt with sulfone derivatives containing an 1, 3, 4-oxadiazole moiety. Journal of agricultural and food chemistry. 2012 Jan 19;60(4):1036-41.

23. Zhang XM, Qiu M, Sun J, Zhang YB, Yang YS, Wang XL, Tang JF, Zhu HL. Synthesis, biological evaluation, and molecular docking studies of 1, 3, 4-oxadiazole derivatives possessing 1, 4-benzodioxan moiety as potential anticancer agents. Bioorganic \& medicinal chemistry. 2011 Nov 1;19(21):6518-24. 